\title{
Review Article \\ Oxidative Stress Relevance in the Pathogenesis of the Rheumatoid Arthritis: A Systematic Review
}

\author{
Celia María Quiñonez-Flores, ${ }^{1,2}$ Susana Aideé González-Chávez, ${ }^{1,2}$ \\ Danyella Del Río Nájera, ${ }^{2}$ and César Pacheco-Tena ${ }^{2}$ \\ ${ }^{1}$ Facultad de Ciencias de la Cultura Física, Universidad Autónoma de Chihuahua, Circuito No. 1,
Nuevo Campus Universitario, Apartado Postal 1552, 31240 Chihuahua, CHIH, Mexico
${ }^{2}$ Facultad de Medicina y Ciencias Biomédicas, Universidad Autónoma de Chihuahua, Circuito No. 1,
Nuevo Campus Universitario, Apartado Postal 1552, 31240 Chihuahua, CHIH, Mexico
}

Correspondence should be addressed to César Pacheco-Tena; dr.cesarpacheco@gmail.com

Received 27 November 2015; Revised 15 March 2016; Accepted 3 April 2016

Academic Editor: Kota V. Ramana

Copyright (c) 2016 Celia María Quiñonez-Flores et al. This is an open access article distributed under the Creative Commons Attribution License, which permits unrestricted use, distribution, and reproduction in any medium, provided the original work is properly cited.

\begin{abstract}
Rheumatoid arthritis (RA) is an autoimmune inflammatory disease whose pathogenic mechanisms remain to be elucidated. The oxidative stress and antioxidants play an important role in the disease process of RA. The study of oxidants and antioxidants biomarkers in RA patients could improve our understanding of disease pathogenesis; likely determining the oxidative stress levels in these patients could prove helpful in assessing disease activity and might also have prognostic implications. To date, the usefulness of oxidative stress biomarkers in RA patients is unclear and the evidence supporting them is heterogeneous. In order to resume and update the information in the status of oxidants and antioxidants and their connection as biomarkers in RA, we performed a systematic literature search in the PubMed database, including clinical trials published in the last five years using the word combination "rheumatoid arthritis oxidative stress". In conclusion, this review supports the fact that the oxidative stress is an active process in RA pathogenesis interrelated to other better known pathogenic elements. However, some controversial results preclude a definite conclusion.
\end{abstract}

\section{Introduction}

Rheumatoid arthritis (RA) is an autoimmune disease affecting diarthrodial joints. It is characterized by erosive synovitis, which causes cartilage and bone destruction and systemic complications including cardiovascular, pulmonary, psychological, and other skeletal disorders [1]. Several autoantibodies have been associated with RA such as rheumatoid factor (RF) and anti-citrullinated protein antibodies (ACPA). RA significantly decreases patients' functional capacity, increases the morbidity and mortality rates, and results in significant costs for the health and social care systems [2]. The prevalence of RA is $1 \%$ of the worldwide population and women are more affected. Although the onset is more frequent during the fourth and fifth decades of life, RA can occur at any age [3]. The etiology and pathogenesis of this disease remain unresolved. It is thought that interactions among various factors, including genetic and environmental factors, lead to an inappropriate immunomodulation and result in an inflammatory process resulting in the damage of synovial structures [1]. Regardless of the exact trigger, the reactive oxygen species (ROS) have been implicated to play an important role in this process [4].

ROS are the most important class of radicals generated in living systems. They are oxygen-derived radicals and include the superoxide radical $\left(\mathrm{O}_{2}^{-{ }^{-}}\right)$, peroxyl radical $\left(\mathrm{ROO}^{\circ}\right)$, perhydroxyl radical $\left(\mathrm{HO}_{2}{ }^{\circ}\right)$ and hydroxyl radical $\left({ }^{\circ} \mathrm{OH}\right)$, and non-free radical species such as hydrogen peroxide $\left(\mathrm{H}_{2} \mathrm{O}_{2}\right)$ and singlet oxygen $\left({ }^{1} \mathrm{O}_{2}\right)$ that are easily converted into free radicals. Nitric oxide $\left(\mathrm{NO}^{*}\right)$, nitrogen dioxide $\left(\mathrm{NO}_{2}{ }^{\circ}\right)$, and peroxynitrite $\left(\mathrm{OONO}^{-}\right)$represent the most important Reactive Nitrogen Species (RNS) [5]. These chemical species contain one or more unpaired electrons in the outermost orbital shell and are called free radicals [6]. They are unstable, 


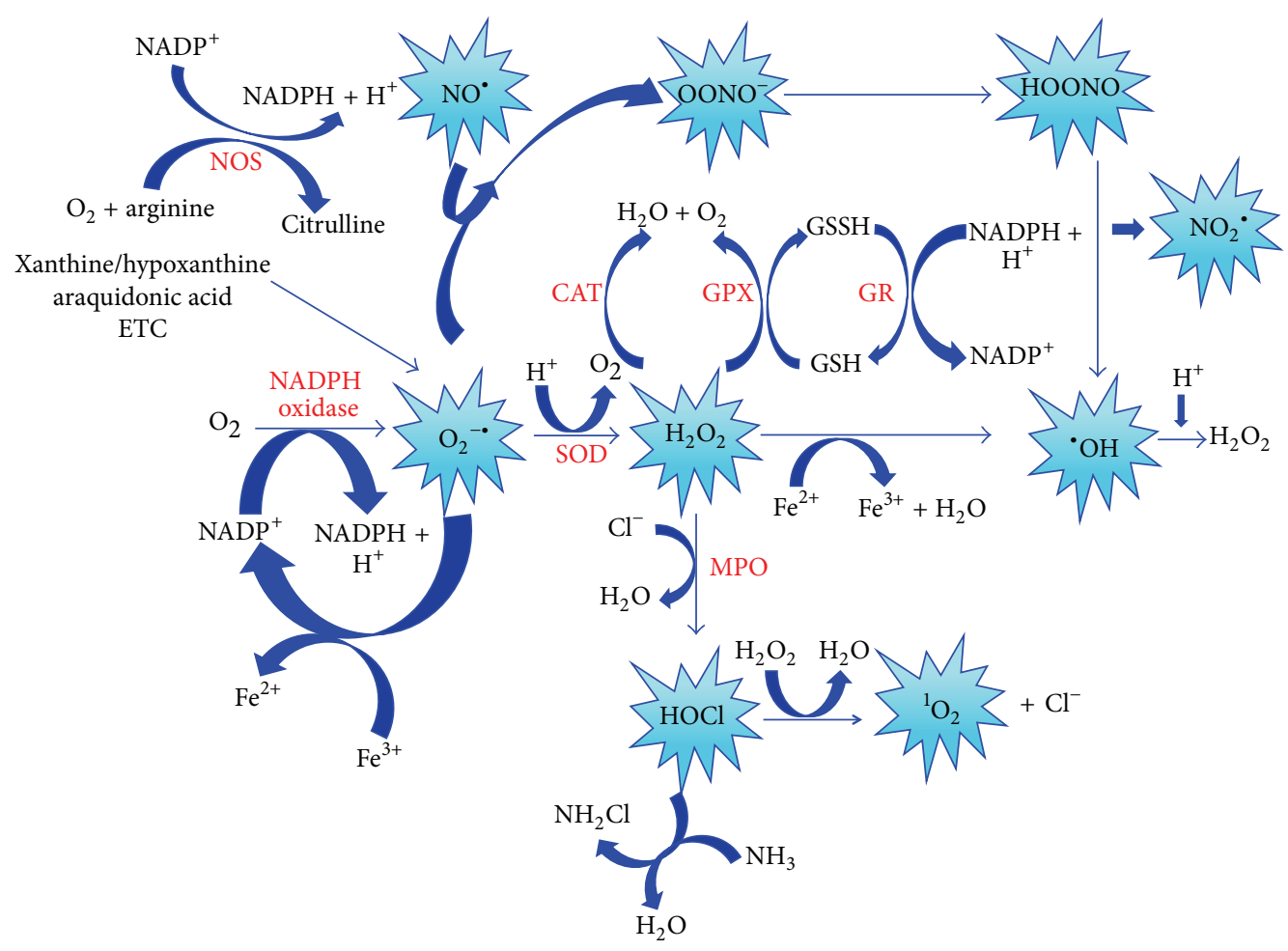

FIGURE 1: Generation of oxygen and nitrogen reactive species (ROS and RNS). CAT: catalase, ETC: electron transport chain, $\mathrm{H}_{2} \mathrm{O}:$ water, $\mathrm{H}_{2} \mathrm{O}_{2}$ : hydrogen peroxide, $\mathrm{HOCl}$ : hypochlorous acid, HOONO: peroxynitrous acid, GPx: glutathione peroxidase, GR: glutathione reductase, GSH: reduced glutathione, GSSH: oxidized glutathione, MPO: myeloperoxidase, NADPH: reduced nicotinamide adenine dinucleotide phosphate, NOS: nitric oxide synthase, $\mathrm{NH}_{2} \mathrm{Cl}$ : chloramine, $\mathrm{NH}_{3}$ : ammonia, $\mathrm{NO}{ }^{\circ}$ : nitric oxide, $\mathrm{NO}_{2}{ }^{\circ}$ : nitrogen dioxide, $\mathrm{O}_{2}$ : oxygen, ${ }^{1} \mathrm{O}_{2}$ : singlet oxygen, $\mathrm{O}_{2}^{-\bullet}$ : superoxide anion, ${ }^{\circ} \mathrm{OH}$ : hydroxyl radical, $\mathrm{OONO}^{-}$: peroxynitrite, and SOD: superoxide dismutase.

highly reactive and short-lived. Free radicals can abstract electrons from other compounds to attain stability; thus the attacked molecule loses its electron and becomes a free radical itself, beginning a chain reaction cascade [5]. The ROS and RNS generation processes are represented in Figure 1.

Under physiological conditions, ROS are required to maintain the cell redox state and play a role in cell signaling, differentiation, proliferation, growth, apoptosis, cytoskeletal regulation, and phagocytosis. However if the concentrations of ROS are increased beyond physiological conditions they can damage cellular components, such as the lipids in the cell membranes, and also proteins and nucleic acids. If a given condition induces an imbalance between oxidants and antioxidants, where oxidants are favored, a disruption of redox signaling is produced, and a control and/or molecular damage occurs. This cellular state termed oxidative stress [4] can result from an excess of oxidants, antioxidants deficiency, or both conditions [7].

The damaging effect of free radicals is counteracted by the action of antioxidants. An antioxidant is any substance or compound capable to scavenge free radicals or inhibiting the oxidation process in the cell [8]. Enzymatic antioxidant response is carried out by superoxide dismutase (SOD), catalase (CAT), and glutathione (GSH) related enzymes (glutathione peroxidase [GPx], glutathione reductase [GR], and thioredoxin reductase). Furthermore, the nonenzymatic antioxidant response includes the action of vitamins
(A, C, and E), $\beta$-carotene, antioxidant minerals (copper, ferritin, zinc, manganese, and selenium) and L- $\gamma$-glutamyl-Lcysteinylglycine (GSH), which is the most important nonenzymatic antioxidant defense $[4,9]$.

$\mathrm{RA}$ is one of the conditions that induce oxidative stress. A fivefold increase in mitochondrial ROS production in whole blood and monocytes of RA patients-compared with healthy subjects-suggests that oxidative stress is a pathogenic hallmark in RA. Free radicals are indirectly implicated in joint damage because they also play an important role as secondary messengers in inflammatory and immunological cellular response in RA. T-cell exposure to increased oxidative stress becomes refractory to several stimuli including those for growth and death and may perpetuate the abnormal immune response [10]. On the other hand, free radicals can degrade directly the joint cartilage, attacking its proteoglycan and inhibiting its synthesis [11]. Oxidative damage of hyaluronic acid and lipoperoxidation products and oxidation of low-density lipoproteins and carbonyl increment resulting from protein oxidation have also been demonstrated in RA as well as DNA damage. ROS-induced genotoxic events have also been linked to mutation of p53 in RA-derived fibroblast-like synoviocytes [9]. Furthermore, it has been suggested that antioxidants systems, either enzymatic or not, are impaired in RA. Low levels of GSH [12], tocopherols, $\beta$-carotene, and retinols and low activities of GR and SOD have been associated [13]. 
The chronic oxidative stress in the RA synovium has been explained by the elevated intra-articular pressure in RA joints, which increases ROS production in the cellular oxidative phosphorylation and induces repetitive cycles of hypoxia/reoxygenation. The hypoxia is an event observed in RA joints whose origin has been explained to be a consequence of the rapid cellular proliferation induced by the inflammatory response; however, according Jeon et al. [14], the hypoxia precedes inflammation at least in an animal arthritis model. From the "Danger Model" point of view, in which the synoviocyte is an impaired cell, this sequence of events could be happening in the human disease [15]. Activated phagocytic cells can also enhance this oxidative stress during oxidative burst. Environmental factors such smoking, drugs, and ultraviolet light may also play a role.

The association between oxidative stress and RA has been explored using various oxidant or antioxidant biomarkers. These biomarkers include lipids, proteins, and DNA oxidation markers and also levels of enzymatic activities, antioxidants agents, and even the direct measurement of free radicals. The aim of this review is to resume and update the available evidence in regard to the potential role of oxidants and antioxidants in RA patients and the findings related to these biomarkers in RA. A systematic literature search was performed including studies published in the last 5 years. The findings are presented in a comparative way between studies included.

\section{Methods}

A systematic literature search was performed including studies published between May 2010 and May 2015. These studies assessed oxidant and/or antioxidant biomarkers in RA patients. The search was conducted in the PubMed database. The word combination used for the search was "rheumatoid arthritis oxidative stress". The article selection was performed using the inclusion and exclusion criteria described below.

2.1. Selection Criteria. This review included original articles published in English within the last 5 years. The studies were clinical trials, which assessed oxidant and/or antioxidant biomarkers in RA patients. Even if the main purpose of any of the selected articles was to compare different diseases, we selected only the results obtained from RA. Studies with experimental interventions and those with oxidative stress induced by causes other than RA (periodontitis, smoking, nutritional status, and genetic polymorphisms in RA) were excluded.

2.2. Methodological Quality. We assessed the methodological quality of the included studies with the Newcastle-Ottawa Quality Assessment Scale (NOS). Every study received a score consisting in a number of stars. The NOS include three domains: (a) selection (maximum 4 stars), (b) comparability (maximum 2 stars), and (c) exposure (maximum 3 stars). The highest score possible was 9 stars. Studies with scores of 6 stars or above were considered to be of moderate to good study quality. The score was not an exclusion criterion. The quality of the selected articles was assessed by one reviewer and checked by a second reviewer.

2.3. Analysis of Information. We selected the following information from every included article: age, female/male ratio, sample size, disease activity score (DAS-28), disease duration, type of biological sample, oxidant and antioxidant biomarkers levels, and the findings related to these biomarkers. This information was organized in comparative tables.

\section{Results}

3.1. Selected Articles. The process of article selection is described in Figure 2. From 518 articles, a total of 22 studies fulfilling our inclusion criteria and not the exclusion criteria were selected for this review. Almost all included studies had moderate or high quality. Eight studies were given 8 stars, 9 studies were given 7 stars, 3 studies were given 6 stars, and 1 study was given 4 stars. Datta et al.s [16] study was included in this review; however this article was not evaluated under NOS criteria due it being not a case-control study. We did not find a published systematic review that was related to the oxidative stress in RA in the last 5 years.

3.2. Study Characteristics. The characteristics of the included studies are summarized in Table 1 . The number of participating patients ranged from 20 to 1720 (cases groups) and 10 to 120 (control group) among studies. The ratio of women to men was higher in both cases and control groups of included studies, with the exception of the study of Nakajima et al. [17]. In some studies $[16,18-24]$ the gender information was not fully detailed. The age mean ranged from 24.2 to 63.4 years. Sixteen articles reported the DAS-28 score and/or the disease duration. The mean DAS-28 score ranged from 2.1 to 5.7 and disease duration from 11 months to 25 years. In general, the studies included healthy individuals as a control group, except for the studies reported by Nakajima et al. [17], Ediz et al. [25], and Nzeusseu Toukap et al. [19], in which Diabetes Mellitus (DM), RA anti-ACPA (-), and Osteoarthritis (OA) were included, respectively. Thiele et al. performed one part of their work with healthy individuals and the other in patients with OA as controls. Only the study from Datta et al. [16] did not include a control group.

In regard to the country of the populations included, 5 studies were conducted in India, 4 in Turkey, 2 in Poland, 1 in New Zealand, 1 in Iran, 2 in USA, 1 in Serbia, 1 in Egypt, 1 in Mexico, 1 in Australia, 1 in Japan, 1 in Belgium, and 1 in China.

3.3. Oxidant and Antioxidant Markers Measured in RA. Thirty different oxidant and/or antioxidant markers were analyzed among the selected studies. They were classified in seven groups: (1) lipid peroxidation (4 markers: malondialdehyde [MDA], thiobarbituric acid reactive substances [TBARS], isoprostane [F2-I], and, malondialdehyde-acetaldehyde [MAA], adducts), (2) protein oxidation (4 markers: protein carbonyls [PC], 3-chlorotyrosine [CT], advanced oxidation of protein products [AOPP], and nitrosothiols [RSNO]), (3) DNA damage (2 markers: micronucleus [MN] and DNA stand breaks [DNA sb]), (4) urate oxidation (1 marker: allantoin 


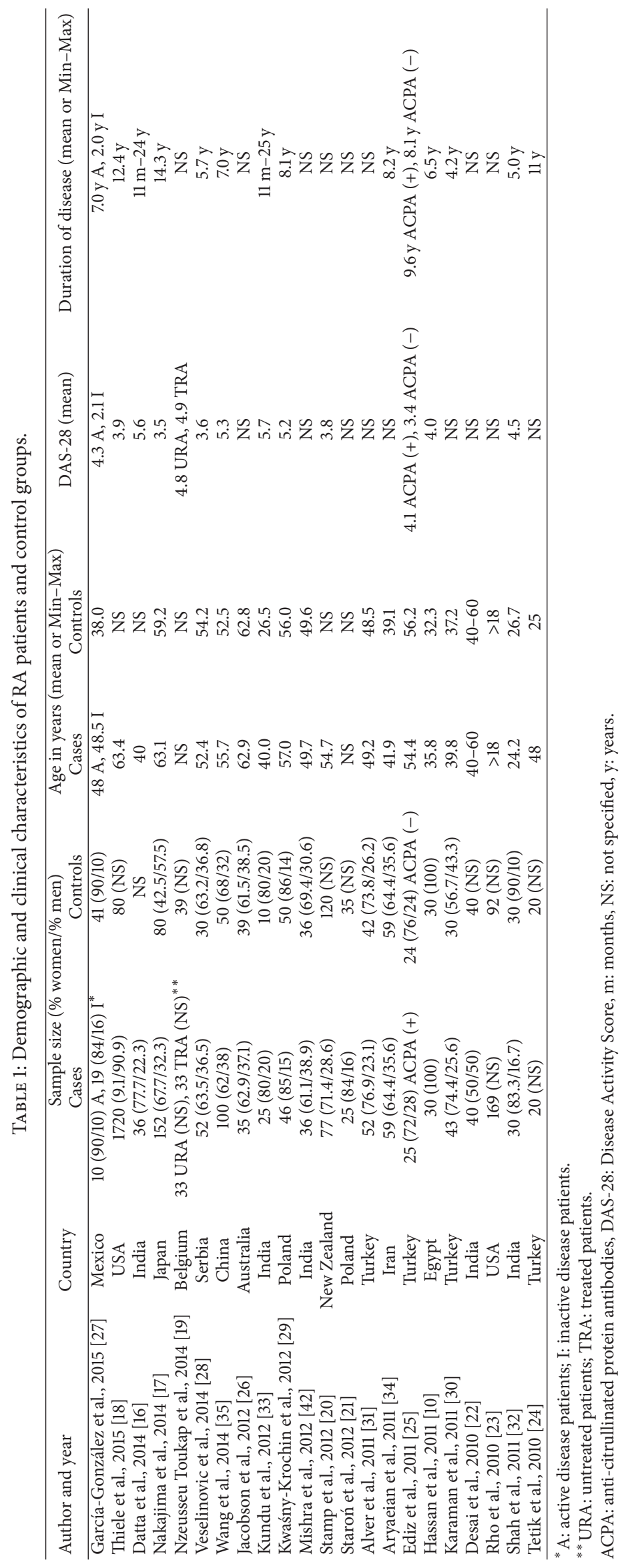




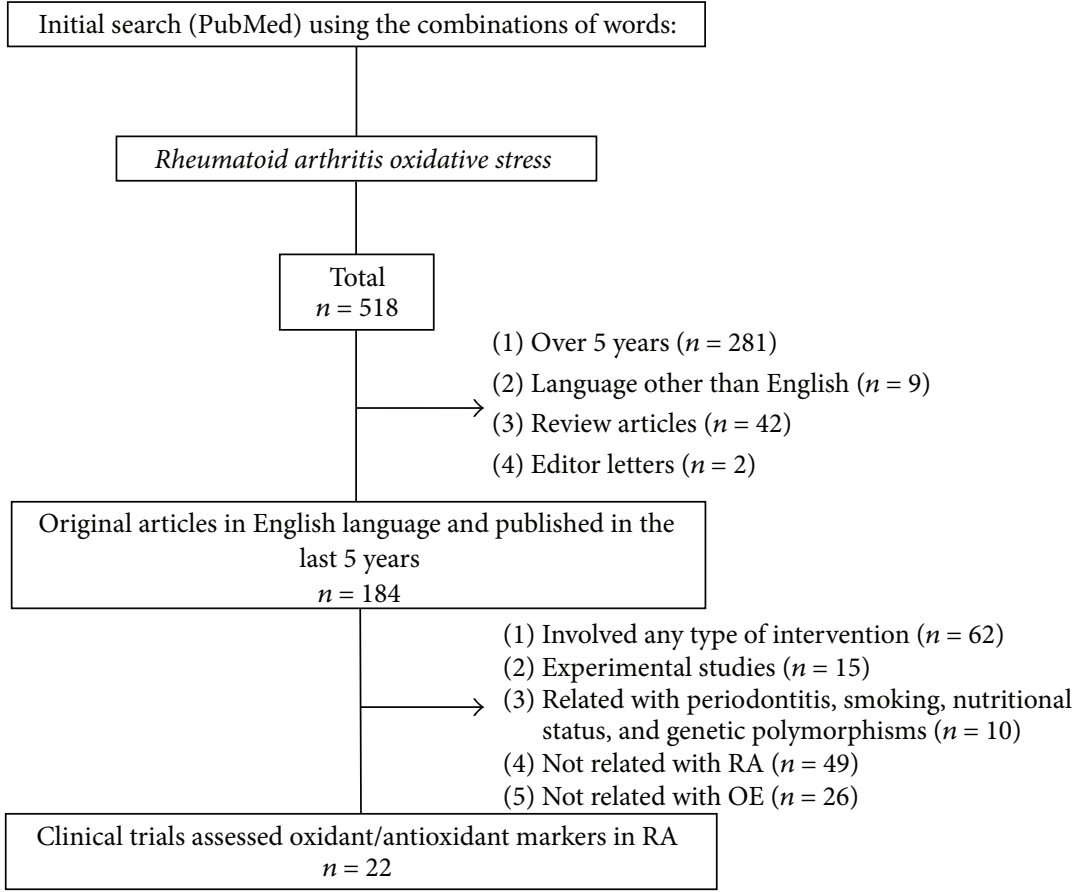

Figure 2: Flow chart of study selection.

[ALLA]), (5) enzymatic activity (7 markers: CAT, SOD, GR, $\mathrm{GPx}$, myeloperoxidase [MPO], NADPH oxidase [NADPH ox], and arylesterase [AE]), (6) antioxidants (6 markers: GSH, oxidized glutathione [GSSG], $\beta$-carotene $[\beta C$ ], vitamin $\mathrm{E}$ [VE], $\mathrm{SH}$ group, and total antioxidant capacity [Anti-Cap]), and (7) free radical/anions (6 markers: total ROS, reactive oxygen metabolites ( $\mathrm{ROM}), \mathrm{H}_{2} \mathrm{O}_{2}, \mathrm{O}_{2}{ }^{-\bullet},{ }^{\bullet} \mathrm{OH}$, and $\mathrm{NO}^{\bullet}$ ) (Table 2). The oxidation of lipids biomarkers was extensively studied (16/22 articles), followed by enzymatic activity $(15 / 22)$, antioxidants $(9 / 22)$, protein oxidation $(5 / 22)$, free radical and anions (4/22), DNA damage (1/22), and uric acid oxidation $(1 / 22)$. The biological samples used in the studies were blood (whole blood, serum, plasma, erythrocytes, and lymphocytes), synovial fluid, synovial tissue, and urine. The blood sample was the most utilized.

3.4. Lipid Oxidation. Sixteen of the 22 studies assessed lipid oxidation biomarkers (MDA, TBARS, F2-I, and MAA adducts). Ten of them measured MDA levels. Most of them observed a statistically significant increase in MDA blood levels in RA patients. A significant difference in MDA blood concentration between RA and control patients was not reported by Jacobson et al. [26] and Ediz et al. [25]; however, Ediz et al., who also measured MDA from RA synovial fluid, reported an MDA increase in this sample. The MDA levels in synovial fluid reported by Datta et al. [16] correlated with levels of both $\mathrm{ROS}$ and ${ }^{\circ} \mathrm{OH}$ radicals and interestingly with the DAS-28 score suggesting an association with disease activity. No study included in this review reported a decrease in MDA levels in patients with RA compared to controls. The blood TBARS levels were measured in 3 studies $[21,27,28]$, which reported a significant increase of these biomarkers in RA.
The F2-I levels were reported in two studies. A significantly higher F2-I excretion in patients with RA than control subjects was found by Rho et al. [23]. This biomarker was associated with a loss of protective effect of HDL cholesterol against coronary calcification. Additionally, plasma levels of F2-I were higher in RA patients compared to controls in the study reported by Kwaśny-Krochin et al. [29]. A positive association between plasma asymmetric dimethylarginine (ADMA) and F2-I and C-Reactive Protein (CRP) concentration in RA samples was reported.

The MAA adducts expression in RA synovial tissue was evaluated only in one study [18] and it was increased in patients with RA if compared to OA. Interestingly, MAA adducts colocalized with citrullinated proteins. Furthermore, increased levels of anti-MAA antibody also correlated to seropositivity for ACPA and RF, suggesting a potential pathogenic role.

3.5. Protein Oxidation. Protein oxidation was evaluated through different biomarkers (PC, RSNO, AOPP, and CT) in 5 studies $[16,19,20,24,27]$. The grade of protein carbonylation was higher in plasma from RA patients if compared with healthy controls in 3 studies [20, 24, 27]. Datta et al. [16] found AOPP protein carbonylation, RSN, and PC present in the synovial fluid from RA patients and also a positive correlation between these biomarkers and DAS-28 score. The level of CT in synovial fluid was also higher in RA patients than in controls [19].

3.6. DNA Oxidation. Only one study assessed the DNA damage by MN and DNAsb (comet assay). Karaman et al. [30] reported DNA damage in RA lymphocytes, in parallel with an 


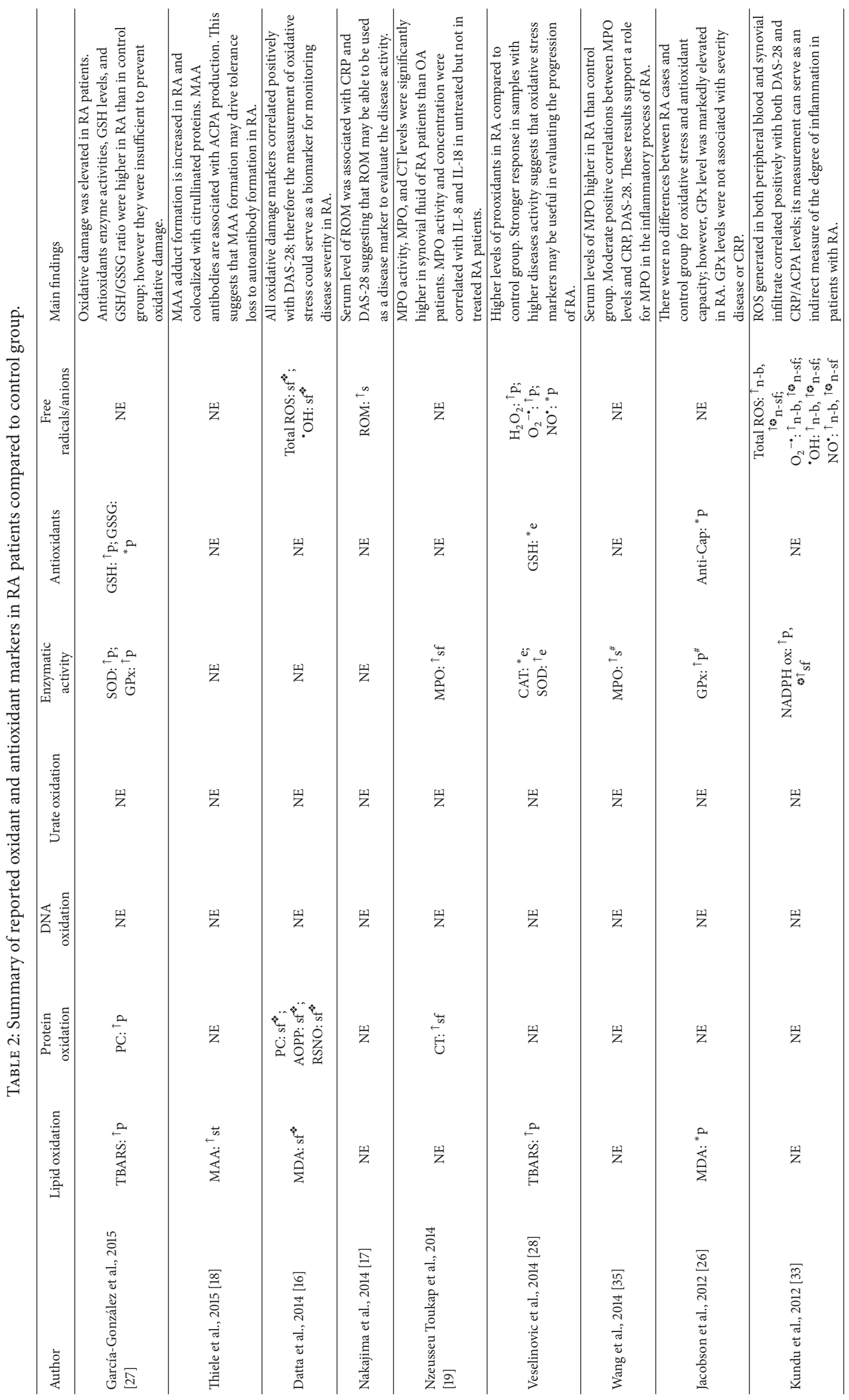




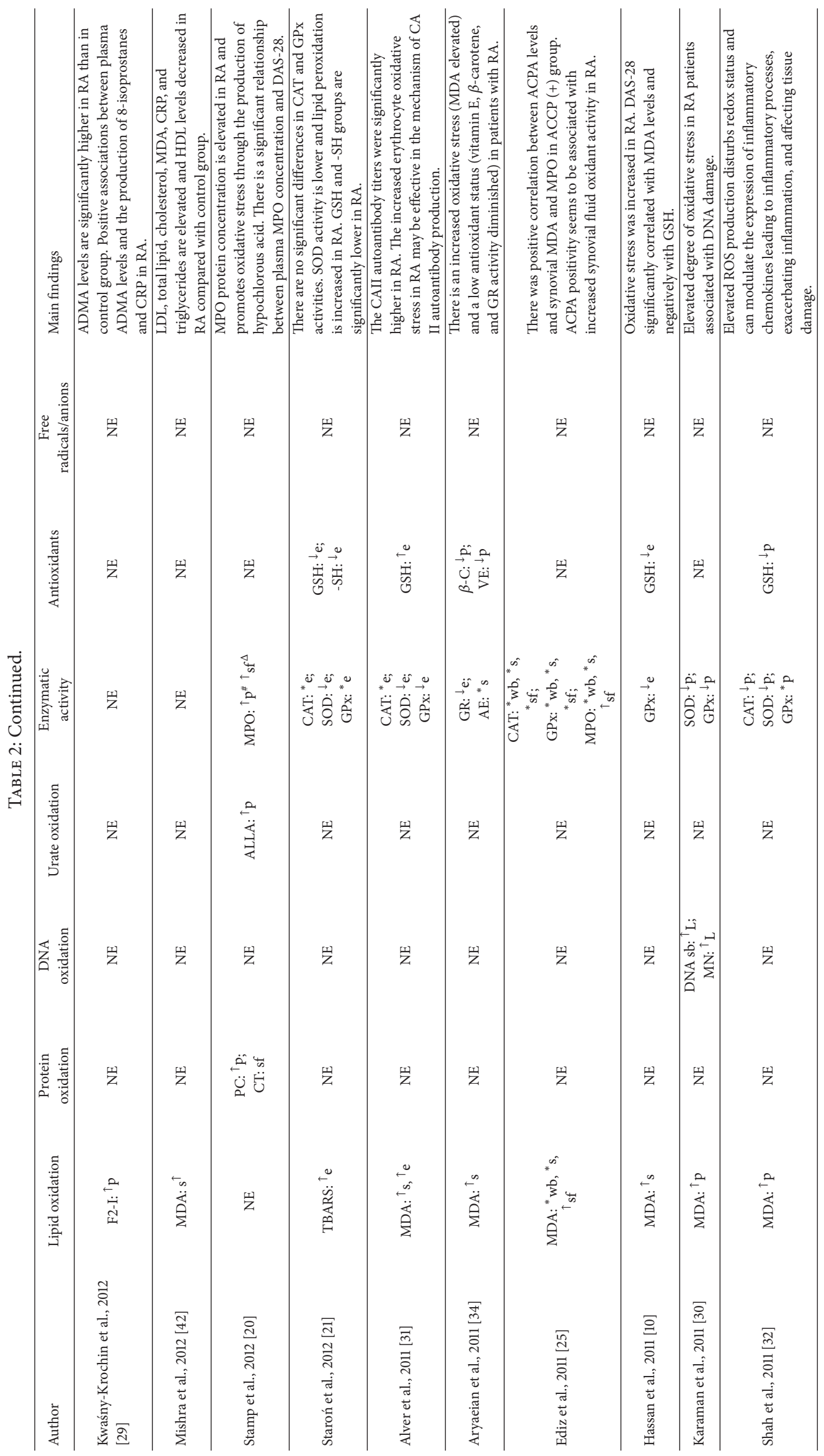




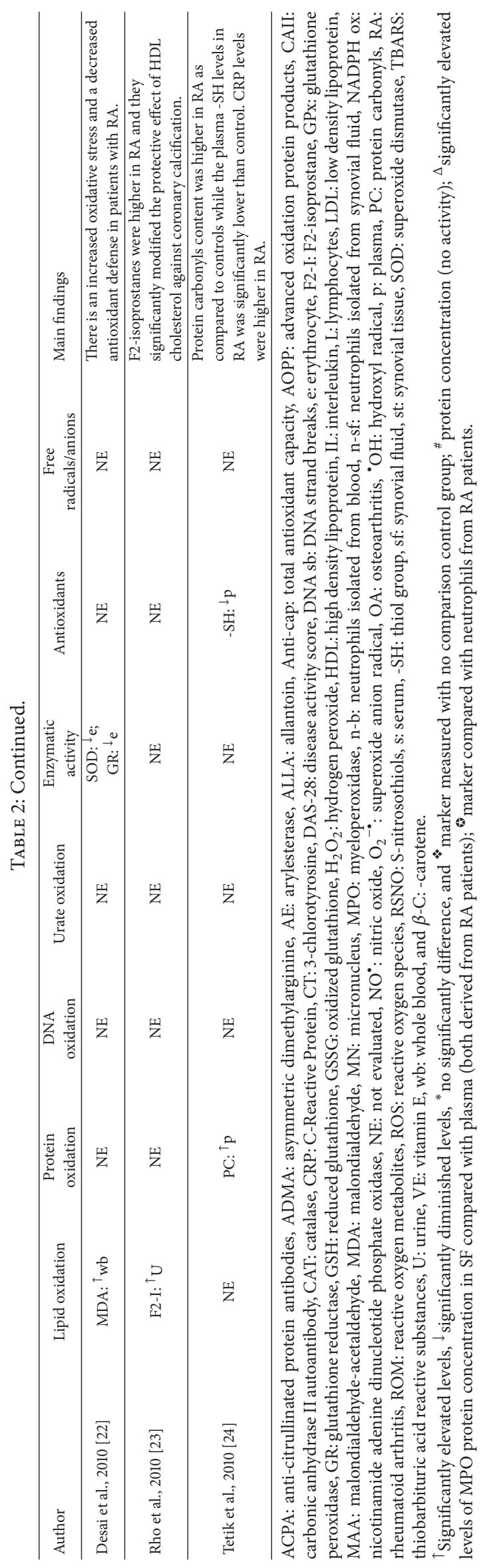


increase of MDA levels and decrease in SOD and GPx activity. These results suggested an increase on oxidative stress in RA, a situation that may impair genetic stability.

3.7. Acid Uric Oxidation. The ALLA plasma concentration as a measure of oxidation of urate was assessed by Stamp et al. [20] and it was higher in RA patients compared with controls. However no correlation with other markers such as MPO or protein carbonyls was found.

3.8. Enzymatic Activity. Enzymatic activity was evaluated in 15 of the included studies (GPx, SOD, CAT, GR, AE, NADPH ox, and MPO); the results were heterogeneous. The enzyme most commonly assessed was GPx (8 studies); it was measured in red blood cells $[10,21,31]$ or in plasma/serum [25-27, 30, 32]. A GPx decreased activity was reported in RA patients in 3 studies [10, 30, 31]; however 2 studies conversely reported an increase on GPx activity in RA patients [26, 27] and 3 more studies did not report any differences between cases and controls in whole blood, serum, synovial fluid, plasma, and erythrocytes $[21,25,32]$. Therefore rendering a conclusion seems complicated at this point.

The SOD activity was also evaluated and gave conflicting results. In 5 studies, the SOD activity from RA patients was found lower than in controls in plasma and erythrocytes [21, 22, 30-32]. Moreover, a higher activity of SOD in RA patients was found in 2 studies [27, 28].

CAT activity was evaluated in 5 studies. Four of these studies [21, 25, 28, 31] did not report any differences between RA patients and controls, whereas the study conducted by Shah et al. [32] showed a lower CAT activity in plasma of RA patients than in healthy controls.

Evidence regarding GR, AE, and NADPH ox activity in RA was limited in the included studies $[22,33,34]$. GR activity was found significantly lower in RA cases than controls [22, 34]; however, AE activity did not show differences between cases and controls [34]. NADPH ox activity was 1.75 -fold higher in RA plasma than healthy individuals. Furthermore, the NADPH activity was significantly higher in RA synovial fluid than in RA peripheral blood [33].

MPO activity was reported in 4 of the included studies. A decrease in the MPO activity in RA plasma was reported by Stamp et al. [20]; however, the activity in synovial fibroblast was found to have increased. Moreover, a significant increase in MPO activity in synovial fluid was reported [19, 25], but not in whole blood and serum [25]. On the other hand, an increase in serum MPO concentration in RA was found in 2 studies $[20,35]$.

3.9. Nonenzymatic Antioxidants. Nine studies assessed different antioxidants molecules. GSH concentrations were measured in 6 studies. With respect to control group, this biomarker was found diminished in 3 studies [10, 21, 32], increased in 2 [27, 31], and unchanged in 1 [28] in RA patients. GSSG levels were evaluated only in one study [27], with no differences between RA patients and the control group. However, the GSH/GSSG ratio was significantly elevated in control group compared to RA patients suggesting that high levels of GSH are associated to the higher GR activity (reduction of GSSH to GSH) in RA compared with controls. $\beta \mathrm{C}$ and VE antioxidants were determined in one study [34] and were lower in RA patients than controls. Likewise, $\mathrm{SH}$ groups were assessed and reported decreased in RA samples for 2 studies [21,24]. Total antioxidant capacity assay was used in one study in which no differences were found between RA and control group [26].

3.10. Free Radicals/Anions. Total ROS, ROM, $\mathrm{H}_{2} \mathrm{O}_{2}, \mathrm{O}_{2}{ }^{-\bullet}$, ${ }^{\circ} \mathrm{OH}$, and $\mathrm{NO}^{\circ}$ were used as oxidative stress biomarkers in 4 studies [16, 17, 28, 33]. All these biomarkers were found elevated in RA patients suggesting an active oxidative process. Only for $\mathrm{NO}^{\bullet}$ contrasting findings were reported among studies. No differences were found in $\mathrm{NO}^{\circ}$ plasma levels between RA and control group [28]; however, $\mathrm{NO}^{\bullet}$ in monocytes from blood of RA patients was higher than control group [33].

\section{Discussion}

The aim of this review was to show and update the available evidence in regard to oxidants and antioxidants in RA patients and to highlight the findings related to biomarkers. Several oxidative stress molecules have been explored as potential biomarkers to monitor the disease progression and explore their role in the RA pathogenesis. Therefore, we consider that it was relevant to review the information in the last 5 years in a systematized approach. To our knowledge, no systematic review that analyzes this set of biomarkers has been published within the last 5 years.

In our revision, the information connecting free radical biomarkers with the oxidative damage was very consistent (Figure 3). In all the studies, total ROS, $\mathrm{H}_{2} \mathrm{O}_{2}, \mathrm{O}_{2}{ }^{-\bullet}$, and ${ }^{\circ} \mathrm{OH}$ were higher in RA patients regardless of the sample used. Interestingly, a new method called d-ROM, which measures ROM in blood, was used in one study [17]. ROM were used as an overall oxidative stress parameter because they are relatively more stable in the blood than direct measurement of ROS. In this study, the ROM concentrations were increased in RA samples compared to controls and correlated with DAS-28 scores and CRP. This information suggests that the use of ROM in conjunction with CRP could be a useful biomarker to evaluate the activity of the disease. These results are consistent with the findings reported by Hayashi et al. [36] in a nutritional study (therefore was not included in this review) where higher levels of ROM in blood and saliva from RA patients also correlated with DAS-28 scores.

Other free radicals biomarkers were analyzed in the included studies. Veselinovic et al. [28] reported significantly higher levels of $\mathrm{O}_{2}^{-\bullet}$ and $\mathrm{H}_{2} \mathrm{O}_{2}$ in RA plasma. The authors suppose that $\mathrm{O}_{2}{ }^{-\bullet}$ radicals in plasma could be dismutated to produce $\mathrm{H}_{2} \mathrm{O}_{2}$ by an upregulated SOD, but CAT or GSH did not detoxify the $\mathrm{H}_{2} \mathrm{O}_{2}$. In the same study, $\mathrm{NO}^{\bullet}$ negatively correlated with GSH, which is a possible compensatory effect of intracellular nonenzymatic antioxidative mechanisms to an increased $\mathrm{NO}_{2}{ }^{-}$production. Kundu et al. [33] found that levels of total $\mathrm{ROS}, \mathrm{O}_{2}{ }^{-\bullet}$, and ${ }^{\bullet} \mathrm{OH}$ radicals were significantly increased in neutrophils from peripheral blood and synovial infiltrate and also showed a strong positive correlation with both DAS-28 and CRP/ACPA levels. Positive correlations of 


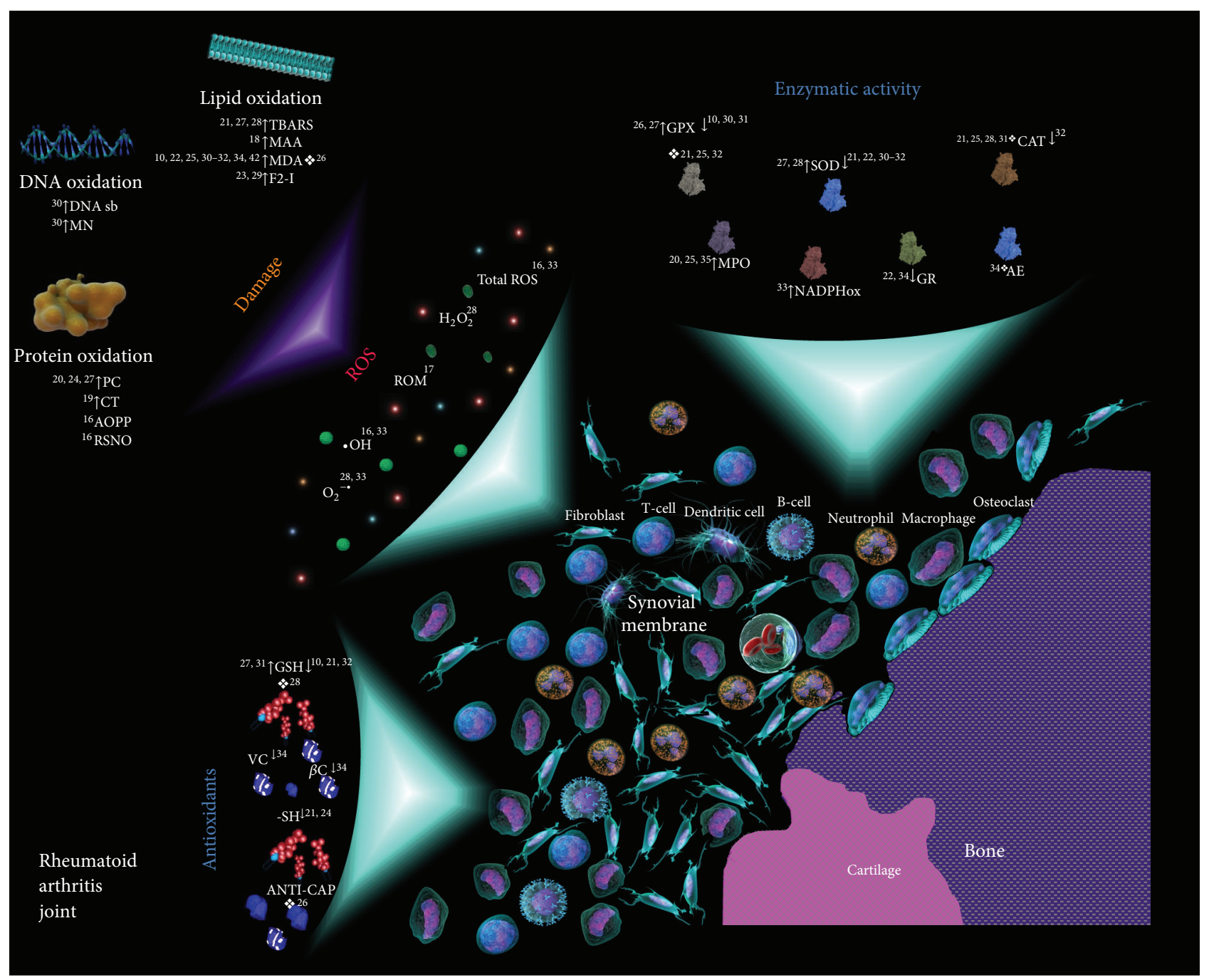

FIGURE 3: Oxidants/antioxidants biomarkers and oxidative damage found in joints and blood of RA patients. The literature references pertaining to the indicated phenomena are provided in the scheme. Under oxidative stress conditions, the joints and blood of patients with RA show high concentrations of free radicals, mainly ROS (gray), which induce DNA, proteins, and lipids damage through different mechanisms. The nonenzymatic antioxidant response (blue square), in general, is diminished. The enzymatic activity (including enzymatic antioxidant response) shows variability. AE: arylesterase, ANTI-CAP: total antioxidant capacity, AOPP: advanced oxidation protein products, CAT: catalase, CT: 3-chlorotyrosine, DNA sb: DNA strand breaks, F2-I: F2-isoprostane, GPx: glutathione peroxidase, GR: glutathione reductase, GSH: reduced glutathione, $\mathrm{H}_{2} \mathrm{O}_{2}$ : hydrogen peroxide, MAA: malondialdehyde-acetaldehyde, MDA: malondialdehyde, MN: micronucleus, MPO: myeloperoxidase, NADPH ox: reduced nicotinamide adenine dinucleotide phosphate oxidase, $\mathrm{O}_{2}^{-\bullet}$ : superoxide anion, ${ }^{\bullet} \mathrm{OH}$ : hydroxyl radical, PC: protein carbonyls, RA: Rheumatoid Arthritis, ROM: reactive oxygen metabolites, ROS: reactive oxygen species, RSNO: Snitrosothiols, -SH: thiol group, SOD: superoxide dismutase, TBARS: thiobarbituric acid reactive substances, VE: vitamin E, and $\beta C$ : $\beta$ carotene. ${ }^{\uparrow}$ Significantly elevated levels, ${ }^{\downarrow}$ significantly diminished levels, and ${ }^{\star}$ no significantly difference.

these free radicals with parameters of disease activity and prognosis suggest that the measurement of these biomarkers in RA patients, combined with current markers, could be useful for monitoring disease activity.

The oxidative damage biomarkers (lipid, proteins, uric acid, and DNA oxidation) were also analyzed, aside from the free radicals. In these studies it was shown that the oxidative damage biomarkers are consistently and significantly higher in RA patients if compared to control individuals; this increment was observed in any sample analyzed (serum, plasma, erythrocytes, urine, synovial fluid, and whole blood).
An oxidative stress environment prevails in $\mathrm{RA}$, which results in the oxidation of biomolecules in this disease.

Lipid peroxidation is one of the major consequences of oxidative stress. It alters the fluidity and permeability of cell membranes and impairs the activity of membranebound enzymes. Lipid peroxidation leads to the production of conjugated diene hydroperoxides and unstable substances, which disintegrate into various bioactive aldehydes such as MDA, 4-hydroxynonenal (HNE), and TBARS [5]. It is known that MDA and HNE can alter protein structures and render them antigenic [37]. In 4 of the included studies, MDA 
values positively correlated with DAS-28 score [10, 16, 32, 33]. Moreover, Alver et al. [31] found increased MDA levels in RA serum and erythrocyte in combination with a negative correlation between carbonic anhydrase (CA) II antibody levels and SOD activity. This evidence is consistent with findings in the SOD knockout mice, in which the elevated oxidative stress in erythrocytes causes anti-CA II antibody production [38]. These results suggest that oxidative stress may enhance the antigenic properties of CA II and promote the production of autoantibodies and also indicate that MDA measurement in RA samples could be an effective option to monitoring the disease activity.

Additionally, in the included studies, the anti-MAA antibodies correlated closely with ACPA [18]. Indeed, the presence of ACPA was associated to increased levels of MDA and MPO in RA synovial fluid levels [25]. It is known that MDA can spontaneously break down and form acetaldehyde (AA). Both MDA and AA are highly reactive aldehydes, and together they modify proteins to produce MDA-AA protein adducts called MAA, which are highly immunogenic $[39,40]$. Presumably, MDA modification of constitutive proteins into RA-related neoantigens could play an important role in the generation of immune responses in RA. Interestingly, MAA adducts colocalize with citrullinated proteins in the inflamed synovial tissue of RA patients, but not in the synovial tissue of $\mathrm{OA}$ patients and, furthermore, the anti-MAA antibody levels are associated with seropositivity for ACPA and RF levels [18]. Positive RA patients represent a subset of RA that is characterized by an aggressive disease, including early and progressive bone erosions [41]. The ACPA are produced at least in part in the synovial structures and can enhance oxidative stress in the joints of ACPA positive RA patients. This increased oxidative activity in synovial fluid may be a factor for accelerated bone erosion seen in ACPA positive RA patients.

The effect of oxidative stress on lipids was analyzed in 3 of the included studies. Kwaśny-Krochin et al. [29] described a positive correlation between ADMA levels and the production of isoprostanes and CRP in RA. Moreover, Mishra et al. [42] found that MDA and CRP correlated positively with cholesterol, and Rho et al. [23] reported that isoprostane excretion and HDL cholesterol concentrations are positively associated with the severity of coronary calcification in patients with RA. It is known that oxidative stress and inflammation might augment dyslipidemia in RA, which are risk factors for cardiovascular disease [42]. Dysregulations of hemostasis and local blood flow in vessels are associated with an altered balance between $\mathrm{NO}^{\bullet}$ and $\mathrm{O}_{2}{ }^{-\bullet}$ in endothelial cells. Indeed, the suppression of endothelial $\mathrm{NO}^{\bullet}$ synthase activity has been considered a hallmark of endothelial injury initiating atherosclerosis [43]. Elevated levels of ADMA, an endogenous inhibitor of NOS, can be detected in RA patients regardless of the presence of cardiovascular disease [44]. The activity of dimethylarginine dimethylaminohydrolase, the key enzyme in ADMA degradation, is downregulated by oxidative stress and TNF-alpha [45], which plays a crucial role in RA [46]. This data suggests that the oxidative stress and the inhibition of NOS by ADMA could play an important role in the pathogenesis of vascular injury in RA.
Another way to assess oxidative damage in RA is throughout its effect on proteins. Free radicals can modify both their structure and functions. The presence of elevated protein carbonyls in RA samples, produced either by direct oxidation of certain amino acids, by a secondary reaction with HNE, or by a glycoxidation reaction [47], suggests a strong oxidative stress state since the carbonyl formation requires high levels of oxidative stress which is detectable in the rheumatoid synovium.

Stamp et al. [20] found elevated levels of protein carbonyls and 3-chlorotyrosine along with an increased MPO activity in RA fluid synovial. Since MPO is the unique human enzyme capable of producing 3-chlotyrosine, the detection of this molecule confirms the production of hypochlorous acid in synovial fluid. Furthermore, the association of protein carbonyls with both MPO and 3-clorotyrosine suggests that hypochlorous acid has a main role in the protein oxidation in this site. Additionally, MPO converts LDL into an isoform that promotes foam cell formation within atherosclerotic plaques and promotes a dysfunctional form of HDL [48]. Elevated levels of MPO in RA and its contribution to oxidative stress provide a potential mechanism for the increase in cardiovascular complications observed in RA patients.

Additionally, in one of the included studies, AOPP, protein carbonyls, and RSNO levels were found elevated in RA synovial fluid and correlated positively with DAS-28. Proteins carbonyls also correlated positively with ROS and - OH radical [16]. AOPPs are considered stable oxidation protein biomarkers and include dityrosine-containing and crosslinking protein products mainly formed during oxidative stress by the reaction of plasma albumin with chlorinated oxidants. Moreover, in vitro studies demonstrated that AOPP induce inflammatory response in fibroblast-like synoviocytes (FLSs) mediated by NADPH oxidase-dependent of NF- $\kappa \mathrm{B}$ activation [49]. Meanwhile, RSNO can disrupt the protein structure and interfere with the catalytic activity of various enzymes. RSNO measure reflects the amount of $\mathrm{NO}^{\circ}$ in adducts with cellular - $\mathrm{SH}$ compounds. This data suggests that measurement of biomarkers of protein oxidation could be used to monitor the severity of the disease in RA patients.

The use of antioxidant molecules as indirect biomarkers of oxidative stress was also included in our revision. Of the antioxidants analyzed in the included studies, the $\beta$ carotene, vitamin $\mathrm{E}$, and $\mathrm{SH}$ group were consistently found decreased. However, the GSH levels were discrepant between studies. These results suggest that the antioxidants systems are impaired in RA. In contrast to these findings, Jacobson et al. [26] did not find a difference in total antioxidant capacity between cases and controls. This opposite finding could be explained by the fact that, in some studies, the oxidants and antioxidant systems are evaluated in a partial and independent way. This evaluation could lead to errors in the interpretation of results, since the total oxidative status cannot be demonstrated. The oxidative stress usually is interpreted as the increase in oxidants or the decrease in antioxidants; however, in this interpretation, the fact that the oxidative stress reflects the final effects of the combined action of oxidant and antioxidant systems is not considered. Indeed, there may be individuals with high levels of oxidant 
molecules but with an efficient antioxidant response, as well as subjects without elevated oxidant concentrations but with a deficient antioxidant response. Therefore, the measurement of total oxidant-antioxidant status is the most valid and reliable way to assess the oxidative stress.

Finally, the activity of antioxidant enzymes (SOD, CAT, $\mathrm{GPx}$, and GR) was evaluated in some of the included studies [10, 21, 25-28, 30-32]. The results from these studies were inconsistent and inconclusive. The antioxidant enzyme activity was found increased, decreased, and even equal in RA patients compared to the control subjects. This high heterogeneity observed among studies makes it difficult to draw clear conclusions. The decrease in antioxidant enzyme activity could be explained by the saturation of the enzymatic antioxidant systems and by the enzymatic inhibition, such as the SOD inhibition by hydrogen peroxide [50]. Particularly, CAT activity was found unchanged; this enzyme does not show significant activity under physiological conditions due to its lower affinity than GPx for $\mathrm{H}_{2} \mathrm{O}_{2}$ but becomes an important enzyme at disease state where concentration of $\mathrm{H}_{2} \mathrm{O}_{2}$ is elevated [51]. Furthermore the use of different methodologies to determine enzyme activity can produce diverse results.

Gathering our results, we can conclude that oxidative stress is a dynamic and complex phenomenon occurring in RA and that is involved in the disease pathogenesis in a complex fashion. Unfortunately, the actual evidence is discrepant in the role of some oxidative stress related molecules. These discrepancies complicate our understanding of the mechanism of oxidative stress implication in RA. The variability and complexity of the regulating mechanisms of oxidative stress in humans, which are associated with genetic, epigenetic, age, gender, and dietary factors, can explain these discrepancies. The results of the present revision suggest the plausibility of several oxidative stress related compounds as potential biomarkers to assess the disease activity and probably prognosis. However, the biomarkers need to be validated in prospective clinical studies. This process implicates the compliance with certain requirements which include (a) a stable product of oxidative stress, not susceptible to artificial induction or loss during storage, (b) that this product can be detectable in the target tissue or a valid surrogate tissue where it causes oxidative modification and damage, (c) that it is present in sufficient and measurable concentrations, (d) that it could be determined by an assay that is specific, sensitive, reproducible, and robust, (e) that this compound should be free of confounding factors from dietary intake, and (f) that it could be measurable within a detection limit of a reliable analytical procedure. Additionally, it is essential to consider relevant clinical factors that could lead to misinterpretation of results such as disease duration, disease activity, its treatment, and even the patient status at the moment of the sample collection are important confounding factors that could affect the interpretation of the assays.

Although our study indicates important aspects of the status of oxidative stress in RA, it is important to highlight some of its limitations. The search strategy used in this review was limited to the findings of the last five years.
Also, the search was conducted in a single database using one set of keywords. Expanding the search criteria the number of articles would increase and possibly allow stronger conclusions. A meta-analysis also would be appropriate.

\section{Competing Interests}

The authors declare that there are no competing interests regarding the publication of this paper.

\section{Acknowledgments}

The authors thank Dr. Luz Helena-Sanin and Angelica Hance for their support in this investigation.

\section{References}

[1] I. B. McInnes and G. Schett, "The pathogenesis of rheumatoid arthritis," The New England journal of medicine, vol. 365, no. 23, pp. 2205-2219, 2011.

[2] C. Lajas, L. Abasolo, B. Bellajdel et al., "Costs and predictors of costs in rheumatoid arthritis: a prevalence-based study," Arthritis Care and Research, vol. 49, no. 1, pp. 64-70, 2003.

[3] A. Gibofsky, "Overview of epidemiology, pathophysiology, and diagnosis of rheumatoid arthritis," The American Journal of Managed Care, vol. 18, no. 13, supplement, pp. S295-S302, 2012.

[4] L. I. Filippin, R. Vercelino, N. P. Marroni, and R. M. Xavier, "Redox signalling and the inflammatory response in rheumatoid arthritis," Clinical and Experimental Immunology, vol. 152, no. 3, pp. 415-422, 2008.

[5] A. Phaniendra, D. B. Jestadi, and L. Periyasamy, "Free radicals: properties, sources, targets, and their implication in various diseases," Indian Journal of Clinical Biochemistry, vol. 30, no. 1, pp. 11-26, 2015.

[6] B. Halliwell and J. M. C. Gutteridge, "Role of free radicals and catalytic metal ions in human disease: an overview," Methods in Enzymology, vol. 186, pp. 1-85, 1990.

[7] M. Valko, D. Leibfritz, J. Moncol, M. T. D. Cronin, M. Mazur, and J. Telser, "Free radicals and antioxidants in normal physiological functions and human disease," International Journal of Biochemistry and Cell Biology, vol. 39, no. 1, pp. 44-84, 2007.

[8] N. I. Krinsky, "Mechanism of action of biological antioxidants," Proceedings of the Society for Experimental Biology and Medicine, vol. 200, no. 2, pp. 248-254, 1992.

[9] C. A. Hitchon and H. S. El-Gabalawy, "Oxidation in rheumatoid arthritis," Arthritis Research and Therapy, vol. 6, no. 6, pp. 265278, 2004.

[10] S. Z. Hassan, T. A. Gheita, S. A. Kenawy, A. T. Fahim, I. M. ElSorougy, and M. S. Abdou, "Oxidative stress in systemic lupus erythematosus and rheumatoid arthritis patients: relationship to disease manifestations and activity," International Journal of Rheumatic Diseases, vol. 14, no. 4, pp. 325-331, 2011.

[11] K. Hadjigogos, "The role of free radicals in the pathogenesis of rheumatoid arthritis," Panminerva Medica, vol. 45, no. 1, pp. 713, 2003.

[12] B. Kalpakcioglu and K. Senel, "The interrelation of glutathione reductase, catalase, glutathione peroxidase, superoxide dismutase, and glucose-6-phosphate in the pathogenesis of rheumatoid arthritis," Clinical Rheumatology, vol. 27, no. 2, pp. 141-145, 2008 . 
[13] M. Q. Hassan, R. A. Hadi, Z. S. Al-Rawi, V. A. Padron, and S. J. Stohs, "The glutathione defense system in the pathogenesis of rheumatoid arthritis," Journal of Applied Toxicology, vol. 21, no. 1, pp. 69-73, 2001.

[14] C. H. Jeon, J.-K. Ahn, J.-Y. Chai et al., "Hypoxia appears at pre-arthritic stage and shows co-localization with early synovial inflammation in collagen induced arthritis," Clinical and Experimental Rheumatology, vol. 26, no. 4, pp. 646-648, 2008.

[15] C. Pacheco-Tena and S. A. Gonzalez-Chavez, "The danger model approach to the pathogenesis of the rheumatic diseases," Journal of Immunology Research, vol. 2015, Article ID 506089, 23 pages, 2015.

[16] S. Datta, S. Kundu, P. Ghosh, S. De, A. Ghosh, and M. Chatterjee, "Correlation of oxidant status with oxidative tissue damage in patients with rheumatoid arthritis," Clinical Rheumatology, vol. 33, no. 11, pp. 1557-1564, 2014.

[17] A. Nakajima, Y. Aoki, Y. Shibata et al., "Identification of clinical parameters associated with serum oxidative stress in patients with rheumatoid arthritis," Modern Rheumatology, vol. 24, no. 6, pp. 926-930, 2014.

[18] G. M. Thiele, M. J. Duryee, D. R. Anderson et al., "Malondialdehyde-acetaldehyde adducts and anti-malondialdehydeacetaldehyde antibodies in rheumatoid arthritis," Arthritis and Rheumatology, vol. 67, no. 3, pp. 645-655, 2015.

[19] A. Nzeusseu Toukap, C. Delporte, C. Noyon et al., "Myeloperoxidase and its products in synovial fluid of patients with treated or untreated rheumatoid arthritis," Free Radical Research, vol. 48, no. 4, pp. 461-465, 2014.

[20] L. K. Stamp, I. Khalilova, J. M. Tarr et al., "Myeloperoxidase and oxidative stress in rheumatoid arthritis," Rheumatology, vol. 51, no. 10, pp. 1796-1803, 2012.

[21] A. Staroń, G. Mąkosa, and M. Koter-Michalak, "Oxidative stress in erythrocytes from patients with rheumatoid arthritis," Rheumatology International, vol. 32, no. 2, pp. 331-334, 2012.

[22] P. B. Desai, S. Manjunath, K. Sumangala, K. Chetana, and J. Vanishree, "Oxidative stress and enzymatic antioxidant status in rheumatoid arthritis: a case control study," European Review for Medical and Pharmacological Sciences, vol. 14, no. 11, pp. 959967, 2010.

[23] Y. H. Rho, C. P. Chung, A. Oeser et al., "Interaction between oxidative stress and high-density lipoprotein cholesterol is associated with severity of coronary artery calcification in rheumatoid arthritis," Arthritis Care and Research, vol. 62, no. 10, pp. 1473-1480, 2010.

[24] S. Tetik, S. Ahmad, A. A. Alturfan et al., "Determination of oxidant stress in plasma of rheumatoid arthritis and primary osteoarthritis patients," Indian Journal of Geo-Marine Sciences, vol. 47, no. 6, pp. 353-358, 2010.

[25] L. Ediz, O. Hiz, H. Ozkol, E. Gulcu, M. Toprak, and M. F. Ceylan, "Relationship between anti-CCP antibodies and oxidant and anti-oxidant activity in patients with rheumatoid arthritis," International Journal of Medical Sciences, vol. 8, no. 2, pp. 139147, 2011.

[26] G. A. Jacobson, S. J. Ives, C. Narkowicz, and G. Jones, "Plasma glutathione peroxidase (GSH-Px) concentration is elevated in rheumatoid arthritis: a case-control study," Clinical Rheumatology, vol. 31, no. 11, pp. 1543-1547, 2012.

[27] A. García-González, R. Gaxiola-Robles, and T. Zenteno-Savín, "Oxidative stress in patients with rheumatoid arthritis," Revista de Investigación Clínica: Organo del Hospital de Enfermedades de la Nutrición, vol. 67, no. 1, pp. 46-53, 2015.
[28] M. Veselinovic, N. Barudzic, M. Vuletic et al., "Oxidative stress in rheumatoid arthritis patients: relationship to diseases activity," Molecular and Cellular Biochemistry, vol. 391, no. 1-2, pp. 225-232, 2014.

[29] B. Kwaśny-Krochin, P. Głuszko, and A. Undas, "Plasma asymmetric dimethylarginine in active rheumatoid arthritis: links with oxidative stress and inflammation," Polskie Archiwum Medycyny Wewnetrznej, vol. 122, no. 6, pp. 270-276, 2012.

[30] A. Karaman, D. N. Binici, and M. A. Melikoĝlu, "Comet assay and analysis of micronucleus formation in patients with rheumatoid arthritis," Mutation Research/Genetic Toxicology and Environmental Mutagenesis, vol. 721, no. 1, pp. 1-5, 2011.

[31] A. Alver, A. Şentürk, H. Çakirbay et al., "Carbonic anhydrase II autoantibody and oxidative stress in rheumatoid arthritis," Clinical Biochemistry, vol. 44, no. 17-18, pp. 1385-1389, 2011.

[32] D. Shah, A. Wanchu, and A. Bhatnagar, "Interaction between oxidative stress and chemokines: possible pathogenic role in systemic lupus erythematosus and rheumatoid arthritis," Immunobiology, vol. 216, no. 9, pp. 1010-1017, 2011.

[33] S. Kundu, P. Ghosh, S. Datta, A. Ghosh, S. Chattopadhyay, and M. Chatterjee, "Oxidative stress as a potential biomarker for determining disease activity in patients with Rheumatoid Arthritis," Free Radical Research, vol. 46, no. 12, pp. 1482-1489, 2012.

[34] N. Aryaeian, M. Djalali, F. Shahram, S. H. Jazayeri, M. Chamari, and S. A. Nazari, "Beta-carotene, vitamin E, MDA, glutathione reductase and arylesterase activity levels in patients with active rheumatoid arthritis," Iranian Journal of Public Health, vol. 40, no. 2, pp. 102-109, 2011.

[35] W. Wang, Z. Jian, J. Guo, and X. Ning, "Increased levels of serum myeloperoxidase in patients with active rheumatoid arthritis," Life Sciences, vol. 117, no. 1, pp. 19-23, 2014.

[36] H. Hayashi, K. Satoi, N. Sato-Mito et al., "Nutritional status in relation to adipokines and oxidative stress is associated with disease activity in patients with rheumatoid arthritis," Nutrition, vol. 28, no. 11-12, pp. 1109-1114, 2012.

[37] B. T. Kurien and R. H. Scofield, "Autoimmunity and oxidatively modified autoantigens," Autoimmunity Reviews, vol. 7, no. 7, pp. 567-573, 2008

[38] Y. Iuchi, F. Okada, K. Onuma et al., "Elevated oxidative stress in erythrocytes due to a SOD1 deficiency causes anaemia and triggers autoantibody production," Biochemical Journal, vol. 402, no. 2, pp. 219-227, 2007.

[39] G. M. Thiele, D. J. Tuma, M. S. Willis et al., "Soluble proteins modified with acetaldehyde and malondialdehyde are immunogenic in the absence of adjuvant," Alcoholism: Clinical and Experimental Research, vol. 22, no. 8, pp. 1731-1739, 1998.

[40] D. J. Tuma, M. L. Kearley, G. M. Thiele et al., "Elucidation of reaction scheme describing malondialdehyde-acetaldehydeprotein adduct formation," Chemical Research in Toxicology, vol. 14, no. 7, pp. 822-832, 2001.

[41] S. Rantapää-Dahlqvist, B. A. W. de Jong, E. Berglin et al., "Antibodies against cyclic citrullinated peptide and IgA rheumatoid factor predict the development of rheumatoid arthritis," Arthritis and Rheumatism, vol. 48, no. 10, pp. 2741-2749, 2003.

[42] R. Mishra, A. Singh, V. Chandra et al., "A comparative analysis of serological parameters and oxidative stress in osteoarthritis and Rheumatoid arthritis," Rheumatology International, vol. 32, no. 8, pp. 2377-2382, 2012.

[43] G. K. Hansson and P. Libby, "The immune response in atherosclerosis: a double-edged sword," Nature Reviews Immunology, vol. 6, no. 7, pp. 508-519, 2006. 
[44] A. Surdacki, J. Martens-Lobenhoffer, A. Wloch et al., "Plasma asymmetric dimethylarginine is related to anticitrullinated protein antibodies in rheumatoid arthritis of short duration," Metabolism, vol. 58, no. 3, pp. 316-318, 2009.

[45] H. Miyazaki, H. Matsuoka, J. P. Cooke et al., "Endogenous nitric oxide synthase inhibitor: a novel marker of atherosclerosis," Journal of Cardiology, vol. 33, no. 2, pp. 105-106, 1999.

[46] S. Van Doornum, G. McColl, and I. P. Wicks, "Accelerated atherosclerosis: an extraarticular feature of rheumatoid arthritis?" Arthritis and Rheumatism, vol. 46, no. 4, pp. 862-873, 2002.

[47] I. Dalle-Donne, R. Rossi, R. Colombo, D. Giustarini, and A. Milzani, "Biomarkers of oxidative damage in human disease," Clinical Chemistry, vol. 52, no. 4, pp. 601-623, 2006.

[48] S. J. Nicholls and S. L. Hazen, "Myeloperoxidase, modified lipoproteins, and atherogenesis," Journal of Lipid Research, vol. 50, supplement, pp. S346-S351, 2009.

[49] S. Zheng, Z.-M. Zhong, S. Qin et al., "Advanced oxidation protein products induce inflammatory response in fibroblast-like synoviocytes through NADPH oxidase -dependent activation of NF- $\kappa$ B," Cellular Physiology and Biochemistry, vol. 32, no. 4, pp. 972-985, 2013.

[50] W. Jira, G. Spiteller, and A. Richter, "Increased levels of lipid oxidation products in low density lipoproteins of patients suffering from rheumatoid arthritis," Chemistry and Physics of Lipids, vol. 87, no. 1, pp. 81-89, 1997.

[51] B. Chance, H. Sies, and A. Boveris, "Hydroperoxide metabolism in mammalian organs," Physiological Reviews, vol. 59, no. 3, pp. 527-605, 1979. 


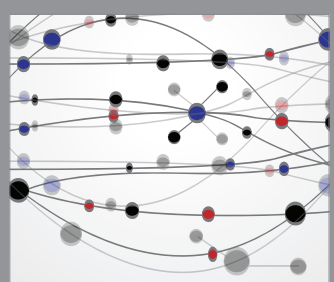

The Scientific World Journal
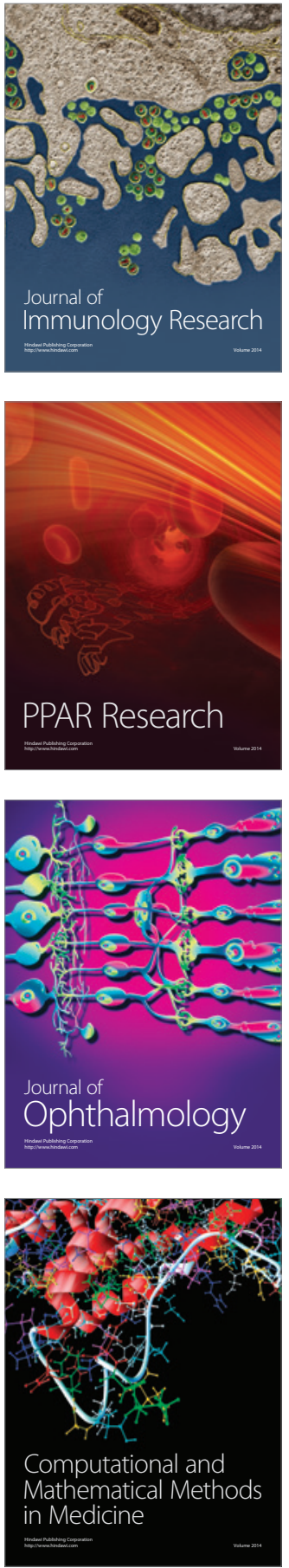

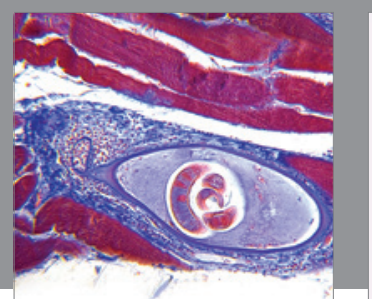

Gastroenterology Research and Practice

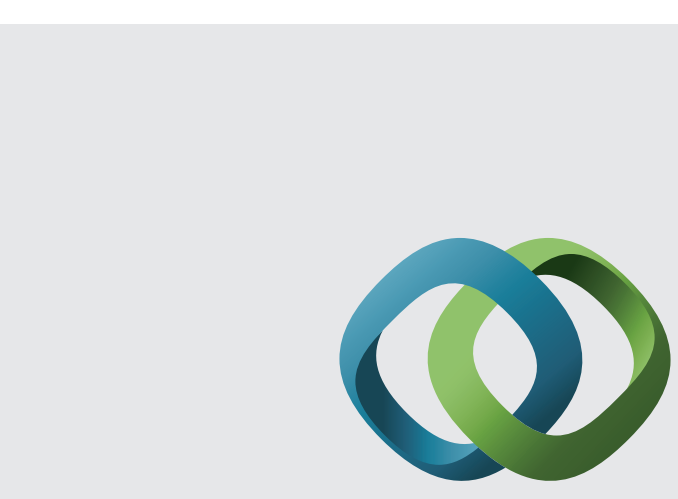

\section{Hindawi}

Submit your manuscripts at

http://www.hindawi.com
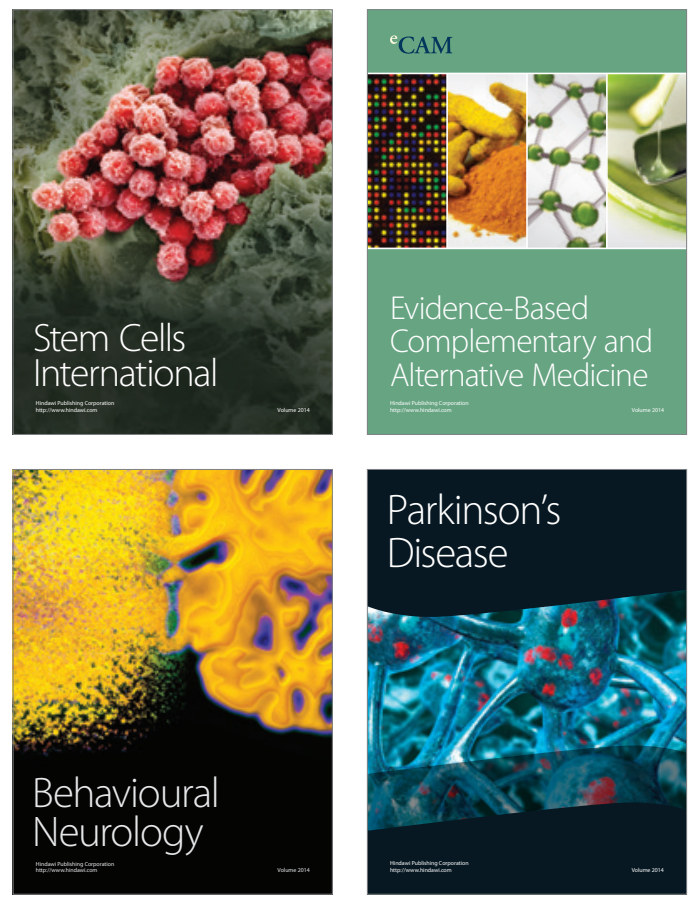
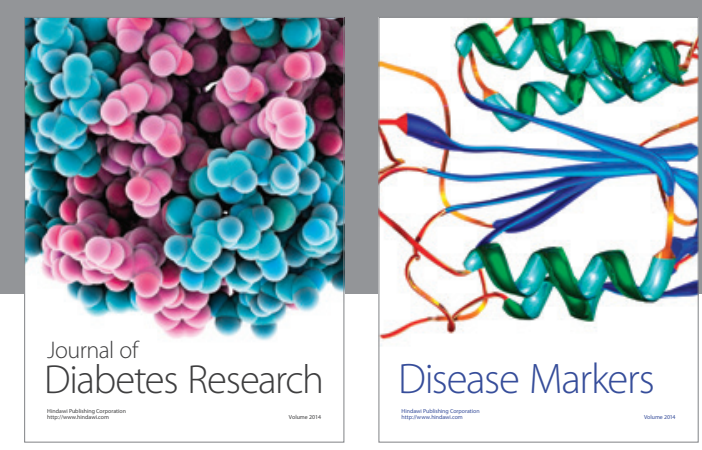

Disease Markers
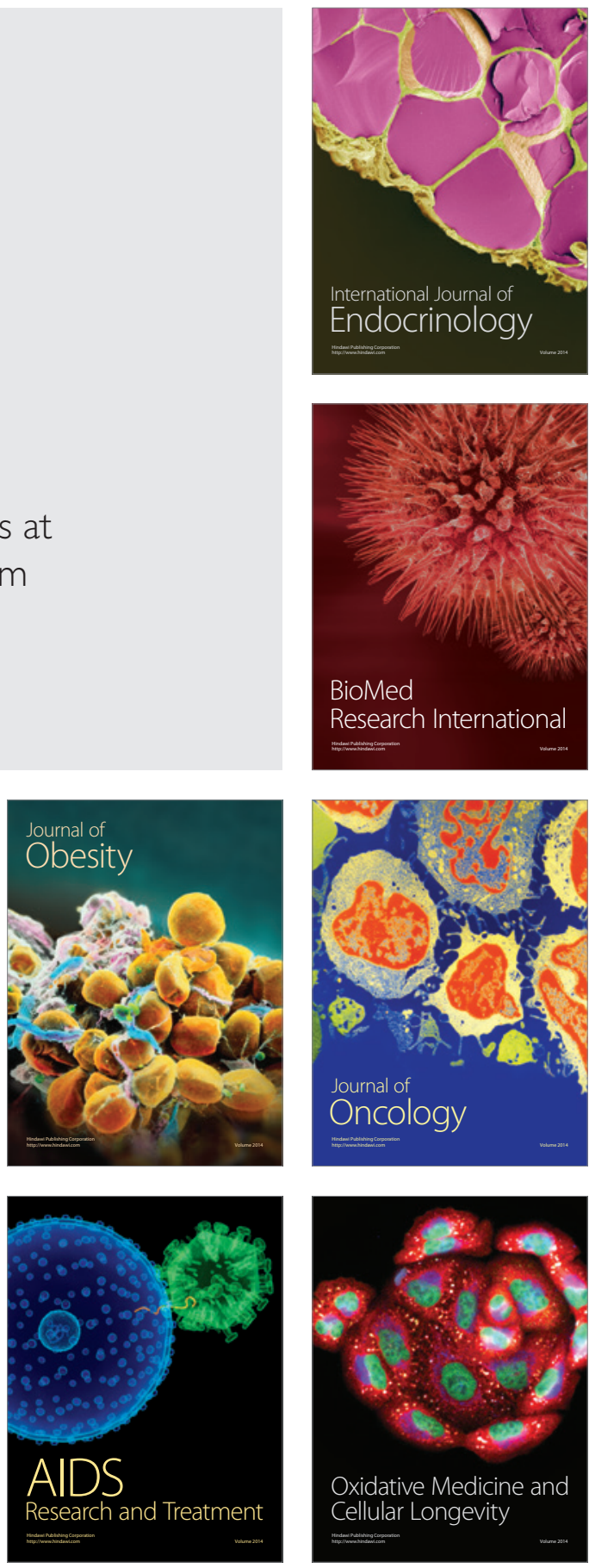